\title{
The Associations of Borderline Personality Disorder Symptoms, Five-Factor Model Personality Dimensions, and Personality Fragmentation among Depressed Inpatients
}

\author{
Timo Säämänen ${ }^{1,2}$, Juha Voutilainen ${ }^{3}$, Jari Lahti ${ }^{1,4,5}$, Erkki Isometsä ${ }^{6,7}$, Martti Heikkinen ${ }^{7}$ and Marius Lahti ${ }^{1,8^{\star}}$ \\ ${ }^{1}$ Institute of Behavioural Sciences, University of Helsinki, Helsinki, Finland \\ ${ }^{2}$ Department of Psychiatry, Hospital District of Helsinki and Uusimaa, Järvenpää, Finland \\ ${ }^{3} \mathrm{Helsinki}$ Department of Social Services and Health Care, Aurora Hospital, Helsinki, Finland \\ ${ }^{4}$ Folkhälsan Research Center, Helsinki, Finland, Topeliusgatan 20, 00250 Helsingfors, Finland \\ ${ }^{5} \mathrm{Helsinki}$ Collegium for Advanced Studies, University of Helsinki, Helsinki, Finland \\ ${ }^{6}$ Institute of Clinical Medicine, University of Helsinki, Helsinki, Finland \\ ${ }^{7}$ Department of Psychiatry, University of Helsinki and Helsinki University Hospital, Helsinki, Finland \\ ${ }^{8}$ Endocrinology Unit, University/BHF Centre for Cardiovascular Science, Queen's Medical Research Institute, University of Edinburgh, Edinburgh, UK
}

\begin{abstract}
Background: Major depressive disorder and borderline personality disorder are highly comorbid. In previous studies, certain Five-Factor Model personality dimensions have been associated with both borderline personality disorder and major depression. Multiple Self States Model personality fragmentation has also been associated with borderline personality disorder. However, the specificity of these associations remains unclear. We examined whether personality fragmentation and Five-Factor Model personality dimensions are associated with borderline personality disorder symptoms among depressed psychiatric patients.
\end{abstract}

Methods: A sample of 43 depressed psychiatric hospital inpatients filled in the Personality Structure Questionnaire, the NEO Personality Inventory, and the Structured Clinical Interview for DSM-IV Axis II Personality Questionnaire on borderline personality disorder symptoms. We examined the associations of Five-Factor Model personality dimensions and Multiple Self States model personality fragmentation with borderline personality disorder symptom sum-score and with the number of endorsed Diagnostic and Statistical Manual of Mental Disorders borderline personality disorder symptoms with linear regression analyses. The analyses were adjusted for age and education level of the participants and/or concurrent depressive symptoms.

Results: Higher personality fragmentation and higher Neuroticism were associated with significantly higher borderline personality disorder symptom sum-score and with a significantly higher number of endorsed borderline personality disorder symptoms. These associations were independent of sociodemographic covariates and concurrent depressive symptoms.

Conclusions: Among depressed patients, higher levels of borderline personality disorder symptoms show independent, significant associations with higher personality fragmentation and higher Neuroticism. These two personality dimensions thus informatively characterize depressed patients with high levels of borderline personality disorder symptoms.

Keywords: Borderline personality disorder; Depression; Neuroticism; Agreeableness; Multiple self-states model

List of Abbreviations: MDD: Major Depressive Disorder; BPD: Borderline Personality Disorder; PSQ: Personality Structure Questionnaire; FFM: Five-Factor Model of personality; SCID IIPQ: Structured Clinical Interview for DSM-IV Axis II Personality Questionnaire; BDI-II: Beck Depression Inventory II; MDQ: Mood Disorder Questionnaire; DSM: Diagnostic and Statistical Manual of mental disorders; NEO-PI: NEO Personality Inventory

\section{Introduction}

Both major depressive disorder (MDD) and borderline personality disorder (BPD) include symptoms of extensively depressed mood and increased suicidality [1]. MDD and BPD are also highly comorbid with each other; BPD is comorbid in 7-30\% of MDD patients [2,3] and MDD is found within $35-74 \%$ of BPD patients [4,5]. Among MDD patients, comorbid BPD is associated with poorer prognosis [6-9] and with an increased healthcare burden $[10,11]$. The high co-occurrence and overlap of the disorders leads us to ask, which are the key characteristics related to BPD within a depressed population? Since dimensional assessment of personality has become a part of new diagnostic systems, individual variations in the distributions of personality dimensions offer one plausible answer to this question.

The Five-Factor Model of Personality (FFM) is the most commonly used personality theory in research and the most extensively validated one [12-14]. The associations of FFM personality dimensions with MDD and BPD have often been assessed and meta-analyses on these associations have been conducted. One meta-analysis found that higher Neuroticism and lower Extraversion were associated with mood disorders [15]. Another meta-analysis showed that especially MDD

*Corresponding author: Marius Lahti, Post-doctoral Researcher, University of Helsinki, Institute of Behavioral Sciences, Siltavuorenpenger $1 \mathrm{~A}$, Helsinki, Uusimaa 00014, Finland, Tel: +358503440662; E-mail: marius.lahti@helsinki.fi

Received December 09, 2015; Accepted April 23, 2016; Published April 30, 2016

Citation: Säämänen T, Voutilainen J, Lahti J, Isometsä E, Heikkinen M, et al. (2016) The Associations of Borderline Personality Disorder Symptoms, Five-Factor Model Personality Dimensions, and Personality Fragmentation among Depressed Inpatients. J Psychiatry 19: 362 doi:10.4172/23785756.1000362

Copyright: (c) 2016 Säämänen T, et al., This is an open-access article distributed under the terms of the Creative Commons Attribution License, which permits unrestricted use, distribution, and reproduction in any medium, provided the original author and source are credited. 
Citation: Säämänen T, Voutilainen J, Lahti J, Isometsä E, Heikkinen M, et al. (2016) The Associations of Borderline Personality Disorder Symptoms, Five-Factor Model Personality Dimensions, and Personality Fragmentation among Depressed Inpatients. J Psychiatry 19: 362 doi: $10.4172 / 2378-5756.1000362$

Page 2 of 8

was associated with higher Neuroticism, lower Extraversion, and also with lower Conscientiousness [16]. In contrast, two independent metaanalyses showed that BPD is associated with higher Neuroticism, lower Agreeableness and lower Conscientiousness [17,18]. Furthermore, the authors know of one study that directly compared FFM profiles in MDD and BPD patients [19]. BPD patients had markedly higher Neuroticism and lower Agreeableness, lower Conscientiousness, and also lower Extraversion scores compared to MDD patients.

On the other hand, BPD patients often move from one reactive mood state to another, experiencing several dysphoric states and periods of euthymia in the course of one day [20], constituting a central difference when comparing BPD to MDD patients. According to the Multiple Self-States Model (MSSM) [21], the shifts in both subjective and interpersonal functioning, mood and the experience of identity that are characteristic of BPD patients can be portrayed as a series of repeating, inflexible and partially dissociated behavioral or emotional patterns, manifesting as self-states. These self-states differ in their level of awareness and co-ordination and in the structural dissociation between them. The MSSM sees the amount of this structural dissociation between self-states as predictive of BPD severity [21-23]. This extent of structural dissociation between self-states is conceptualized as personality multiplicity or personality fragmentation, and it can be measured with the Personality Structure Questionnaire (PSQ) [24]. This level of personality fragmentation has been found to at least partially not correspond with dissociative identity disorder and normal intraindividual variation in emotion and behavior patterns and personality; indicating that this characteristic may be specific to BPD [24,25]. Indeed, previous studies have shown that BPD patients score higher on personality fragmentation than individuals with no mental disorder and patients with dissociative disorder [24] or patients with other personality disorders [25]. In contrast, we know of no studies assessing personality fragmentation levels in MDD.

This study examines whether the FFM personality dimensions and personality fragmentation are associated with BPD symptoms within a depressed psychiatric inpatient population. As stated, these personality dimensions have previously been associated with BPD and/or MDD separately $[15-18,24,25]$ but their specific associations to BPD among depressed patients are unknown. Based on previous findings, we hypothesize that higher Neuroticism, lower Agreeableness and lower Conscientiousness are associated with increased BPD symptoms within a depressed sample. Previous findings on Extraversion are mixed; therefore we make no hypothesis regarding Extraversion. Openness to Experience has not previously been associated with either disorder, and we hypothesize that no association between this personality dimension and BPD symptoms emerge in our study. Although personality fragmentation has not been previously studied in an MDD population, we hypothesize that higher personality fragmentation is associated with increased BPD symptoms also in the current study population. We also examine individual symptom-level associations of specific BPD symptoms with FFM personality dimensions and personality fragmentation. Moreover, the FFM dimensions can be divided into facet scales that according to some evidence may be more informative in depicting personality pathology than the five broader dimensions [17]. Hence, we also examine facet-level associations of three of the FFM personality dimensions with BPD symptoms.

\section{Methods}

The current study sample included 43 inpatients from a psychiatric ward focusing on mood disorder treatments at Aurora Hospital located in Helsinki, Finland. The recruitment of the participants started in December 2009 and ended in May 2011. Patients in this ward have uni- or bipolar mood disorders, are a risk to themselves or others, and are thus taken into a closed hospital ward with varying liberty to leave the ward. All participating patients signed an informed consent form and the study was approved by the Helsinki University Central Hospital ethical board.

We collected data on sociodemographic variables, which included sex, age and the highest attained education level, from self-report questionnaires. The sample comprised 29 (67.4\% of the sample) women and $14(32.6 \%)$ men, and the mean age of the sample was 37.9 years $(\mathrm{SD}=15.5)$. Education level was divided into three categories of "Only elementary school" (23.3\%), "High school or vocational studies" (53.5\%) and "University or applied science degree" (23.3\%).

New patients arriving at the psychiatric ward were screened for depression using the Beck Depression Inventory II (BDI-II) [26] and with the Center for Epidemiological Studies of Depression Scale (CES-D) [27], and for not having any indication of bipolar disorder using the Mood Disorder Questionnaire (MDQ) [28] and asked to participate when suitable. The cut-off score for BDI-II was 20 points or more, which indicates at least moderate depression [26], and the cutoff score for CES-D was 16 points, indicating risk for clinical depression [27]. In MDQ, suspected bipolar disorder was indicated when seven or more items were endorsed, several of the items co-occurred, and when the symptoms caused at least moderate psychosocial impairment [28]. These screening criteria resulted in a sample of patients with self-assessed depression, certified with two validated questionnaires $[26,27]$, from which patients with any suspicion of bipolar disorder were excluded.

BPD symptoms were evaluated using the Structured Clinical Interview for DSM-IV Axis II Personality Questionnaire (SCID IIPQ). This scale shows moderate sensitivity $[29,30]$ and moderate specificity [29] in identifying diagnosis of BPD. It also shows good testretest reliability and high internal consistency [29]. Also in the current sample, the internal consistency for SCID II-PQ was good (Cronbach's $\alpha=0.78$ ).

The SCID II-PQ comprises 15 questions, which the respondent rates on a dichotomous (Yes/No) scale. Each of the SCID-PQ questions assesses one of the BPD symptoms according to Diagnostic and Statistical Manual of Mental Disorders, $4^{\text {th }}$ Edition (DSM-IV) criteria. The SCID-PQ comprises 4 questions on DSM BPD symptom Identity Disturbance, 3 questions on Inappropriate Anger, 2 questions on Suicidal Behavior and 1 question on the symptoms Frantic Efforts to Avoid Abandonment, Unstable Relationships, Impulsivity, Affective Instability, Chronic Feelings of Emptiness and Transient, Stress-Related Paranoid Ideation or Severe Dissociative Symptoms. The BPD symptoms in SCID-II-PQ can be construed either on a scale from 0 to 15 or on a scale from 0 to 9 where each individual DSM BPD symptom is given equal weight. If a certain symptom is assessed by multiple questions, the symptom in question is considered endorsed if the participant responds positively to at least one of the questions on that symptom [30]. While the original sum-score ranging from 0 to 15 is the more traditional approach to use this questionnaire and gives a wider distribution of scores, the 9-item version gives equal weight to each endorsed symptom and thereby corresponds more closely with the DSM criteria for BPD. Hence, we use both the sum-score indicating the amount of endorsed SCID-PQ questions (referred to hereafter as SCID-PQ BPD sum-score) and the amount of endorsed BPD symptoms (referred to as number of endorsed BPD symptoms) as our main outcomes. 
Citation: Säämänen T, Voutilainen J, Lahti J, Isometsä E, Heikkinen M, et al. (2016) The Associations of Borderline Personality Disorder Symptoms, Five-Factor Model Personality Dimensions, and Personality Fragmentation among Depressed Inpatients. J Psychiatry 19: 362 doi:10.4172/2378-5756.1000362

Page 3 of 8

Furthermore, we wanted to shed light on which particular BPD symptoms are associated with FFM personality dimensions and personality fragmentation among depressed inpatients. Hence, we also look at symptom-level associations of the nine BPD symptoms captured in the SCID-PQ.

To assess the FFM personality dimensions, we used the 181-item NESTA self-report questionnaire [14], the Finnish version of the NEO Personality Inventory (NEO-PI) [31]. The scales of Neuroticism, Extraversion, and Openness to Experience comprise 48 items each and those on Agreeableness and Conscientiousness include 18 items. The responses vary between 0 (strongly disagree) and 4 (strongly agree). The five-factor structure of the NEO-PI and its revisions has been replicated in several cultures [13], and has also been found in the Finnish validation sample [14]. In our sample, the reliability of the NEO-PI scales varied from good to excellent, as the Cronbach's alphas were between 0.81 for Agreeableness and 0.92 for Neuroticism.

As stated, in the FFM framework the five FFM dimensions can be divided into personality facets $[13,17]$. However, the original NEOPI version used in the current study comprises facet scales only for three FFM dimensions, Neuroticism, Extraversion and Openness to Experience. Nevertheless, we also examined the associations of BPD symptoms to these NEO-PI facet scale scores: Anxiety, Hostility, Depression, Self-Consciousness, Impulsiveness, and Vulnerability for Neuroticism; Warmth, Gregariousness, Assertiveness, Activity, Excitement-Seeking, and Positive Emotions for Extraversion; and Fantasy, Aesthetics, Feelings, Actions, Ideas, and Values for Openness to Experience [31]. Since the distribution of the Depression scale was skewed to the left, we rank-normalized this scale according to Blom's formula [32].

We used the PSQ as a self-report questionnaire of personality fragmentation $[24,33]$. It contains eight contradictory statement pairs related to within-person variation in personality depicting the amount of fragmentation of self-concept, manifesting as partially dissociated behavioral and emotional patterns. The respondent is asked to evaluate which statement within a contradictory statement pair describes them better on a numerical scale from 1 (Totally true) to 3 (I cannot say) to 5 (Totally true). Sample items include pairs such as "My sense of self is always the same" versus "How I act or feel is constantly changing" and "I have no sense of opposed sides to my nature" versus "I feel I am split between two (or more) ways of being, sharply differentiated from each other". Higher scores indicate higher personality fragmentation. There is overlap between BPD symptoms and PSQ items but PSQ offers a dimensional estimate of structural dissociation and is not restricted to BPD diagnostic criteria, such as identity disturbance or transient, stress-related paranoid ideation or severe dissociative symptoms [1]. Rather, it measures the amount of co-ordination or separation, i.e. structural dissociation between emotional and behavioral patterns [21]. The PSQ has been found to consistently measure a similar construct between items [24,33], and also in our sample its internal consistency was good (Cronbach's $\alpha=0.81$ ).

To facilitate comparison of effect sizes, all the personality dimension variables (PSQ and NEO-PI) and BPD symptom scores (SCID II-PQ BPD sum-score and the number of endorsed BPD symptoms) were standardized. Associations of age, sex, education level and depressive symptoms with BPD symptoms were investigated using Pearson Correlation Analysis, t-tests, and univariate Analysis of Variance (ANOVA). With Pearson Correlation Analysis, we also examined the intercorrelations between the independent variables. To examine which individual BPD symptoms were most associated with FFM personality dimensions and with personality fragmentation, we ran t-test analyses examining group differences between those who endorsed a particular symptom and those who did not. Next, bivariate correlations were calculated for the NEO-PI and PSQ scores with BPD symptoms. Thereafter, all the NEO-PI and PSQ scale scores were individually included in hierarchical multiple regression models as independent variables predicting BPD symptoms. In the first step, we entered the covariates that showed significant associations with BPD symptoms in univariate analyses. In the second step, we entered the independent variable.

\section{Results}

Descriptive statistics of the main study variables are reported in Table 1 and those of FFM facet scales in Supplementary Table 1. On average, the patients reported a high number of BPD symptoms (mean number of endorsed symptoms 5.8 out of 9 possible). Older age was associated with a lower SCID-PQ BPD sum-score $(\mathrm{r}=-0.42, \mathrm{p}=0.01)$ and with a lower number of endorsed BPD symptoms $(\mathrm{r}=-0.35$, $\mathrm{p}=0.02$ ). Patients with only elementary school education had a higher SCID-PQ BPD sum-score than patients with university or applied sciences education [Mean difference (MD) in standard deviation (SD) units $=0.96, p=0.03$ ], but education level was not significantly associated with the number of endorsed BPD symptoms ( $p$-values $\geq 0.08$ ). There were no sex-differences in SCID-PQ BPD sum-score $(\mathrm{MD}=0.50 \mathrm{SDs}$, $\mathrm{p}=0.13$ ) or in the number of endorsed $\mathrm{BPD}$ symptoms ( $\mathrm{MD}=0.55 \mathrm{SDs}$, $\mathrm{p}=0.09$ ).

BDI depressive symptoms were not associated with SCID-PQ BPD sum-score or with the number of endorsed BPD symptoms ( $\mathrm{r}{ }^{`} \mathrm{~s}=0.12$ and $=0.17$, respectively, $p \geq 0.28$ ). CES-D depressive symptoms were neither associated with SCID-PQ BPD symptom sum-score $(\mathrm{r}=0.29$, $\mathrm{p}=0.06$ ). However, higher CES-D depressive symptoms were associated with significantly higher number of endorsed BPD symptoms $(r=0.31$, $\mathrm{p}=0.04)$. Hence, the regression models on SCID-PQ BPD sum-score will include age and education level as covariates, while that on the number on endorsed BPD symptoms will include age and CES-D depressive symptoms as covariates.

Of the independent variables, Neuroticism correlated negatively with Extraversion $(\mathrm{r}=-0.34, \mathrm{p}=0.02)$, Agreeableness $(\mathrm{r}=-0.43, \mathrm{p}=0.004)$, and Conscientiousness $(r=-0.30, p=0.05)$ and positively with PSQ ( $r$ $=0.50, p=0.001)$. Agreeableness correlated negatively with PSQ $(r=$ $0.49, \mathrm{p}=0.001$ ) and Openness to Experience correlated positively with Extraversion $(r=0.38, p=0.01)$.

Table 2 shows the number of patients endorsing a particular BPD symptom and the t-test results on group differences on PSQ personality fragmentation and FFM personality dimensions between patients endorsing a particular BPD symptom and patients without this symptom. Patients with BPD symptoms unstable relationships, identity disturbance, impulsivity, affective instability, inappropriate anger and transient, stress-related paranoid ideation/severe dissociative symptoms scored significantly higher on PSQ personality fragmentation. Patients with BPD symptoms identity disturbance, impulsivity, inappropriate anger and transient, stress-related paranoid ideation/severe dissociative symptoms had significantly higher FFM Neuroticism scores. Patients with affective instability, inappropriate anger, and impulsivity scored significantly lower on Agreeableness. Extraversion scores were significantly higher among patients with affective instability. Conscientiousness and Openness to Experience were not significantly associated with individual BPD symptoms. On the other hand, suicidal behavior and chronic feelings of emptiness 
Citation: Säämänen T, Voutilainen J, Lahti J, Isometsä E, Heikkinen M, et al. (2016) The Associations of Borderline Personality Disorder Symptoms, Five-Factor Model Personality Dimensions, and Personality Fragmentation among Depressed Inpatients. J Psychiatry 19: 362 doi: $10.4172 / 2378-5756.1000362$

Page 4 of 8

\begin{tabular}{|c|c|c|c|}
\hline Assessed Characteristic & Scale & Mean & SD \\
\hline \multirow{2}{*}{ Borderline Personality Disorder Symptoms } & SCID II-PQ Sum-Score & 7.6 & 3.5 \\
\hline & Number of Endorsed BPD Symptoms & 5.8 & 2.3 \\
\hline \multirow{2}{*}{ Depressive Symptoms } & BDI-II & 36.8 & 8.4 \\
\hline & CES-D & 40.7 & 8.2 \\
\hline Personality Fragmentation & $\mathrm{PSQ}$ & 27.1 & 6.4 \\
\hline \multirow{5}{*}{ Five-Factor Model Personality Dimensions } & Neuroticism & 125.8 & 26.6 \\
\hline & Extraversion & 93.7 & 26.6 \\
\hline & Openness to Experience & 127.3 & 24.2 \\
\hline & Agreeableness & 43.3 & 11.1 \\
\hline & Conscientiousness & 38.2 & 11.5 \\
\hline
\end{tabular}

SCID II-PQ: Structured Clinical Interview for DSM-IV Axis II Personality Questionnaire; BDI-II: Beck Depression Inventory II; CES-D: Center for Epidemiological Studies Depression Scale; PSQ: Personality Structure Questionnaire.

Table 1: Means and standard deviations (SD) for Five-Factor Model personality dimensions, personality fragmentation, borderline personality disorder symptoms and depressive symptoms.

\begin{tabular}{|c|c|c|c|c|c|c|c|c|c|c|c|c|c|}
\hline \multirow[b]{2}{*}{ BPD Symptom } & \multirow{2}{*}{$\begin{array}{c}\text { Personality } \\
\text { Dimension } \\
\text { Symptom } \\
\text { Prevalence }\end{array}$} & \multicolumn{2}{|c|}{$\begin{array}{l}\text { Personality } \\
\text { Fragmentation }\end{array}$} & \multicolumn{2}{|c|}{ Neuroticism } & \multicolumn{2}{|c|}{ Extraversion } & \multicolumn{2}{|c|}{$\begin{array}{c}\text { Openness to } \\
\text { Experience }\end{array}$} & \multicolumn{2}{|c|}{ Agreeableness } & \multicolumn{2}{|c|}{ Conscientiousness } \\
\hline & & $\begin{array}{c}\mathrm{MD}^{\mathrm{a}} \\
(95 \% \mathrm{Cl})\end{array}$ & $p$ & $\begin{array}{c}\mathrm{MD}^{\mathrm{a}} \\
(95 \% \mathrm{Cl})\end{array}$ & $p$ & $\begin{array}{c}\mathrm{MD}^{\mathrm{a}} \\
(95 \% \mathrm{Cl})\end{array}$ & $p$ & $\begin{array}{c}M^{\mathrm{a}} \\
(95 \% \mathrm{Cl})\end{array}$ & $p$ & $\begin{array}{c}\mathrm{MD}^{\mathrm{a}} \\
(95 \% \mathrm{Cl})\end{array}$ & $p$ & $\begin{array}{c}\mathrm{MD}^{\mathrm{a}} \\
(95 \% \mathrm{Cl})\end{array}$ & $p$ \\
\hline $\begin{array}{c}\text { Frantic Efforts to Avoid } \\
\text { Abandonment }\end{array}$ & $\begin{array}{c}31 \\
(72.1 \%)\end{array}$ & $\begin{array}{c}0.63 \\
(-0.03-1.30)\end{array}$ & 0.06 & $\begin{array}{c}0.62 \\
(-0.04-1.29)\end{array}$ & 0.07 & $\begin{array}{c}0.45 \\
(-0.23-1.13)\end{array}$ & 0.19 & $\begin{array}{c}-0.30 \\
(-0.99-0.39)\end{array}$ & 0.38 & $\begin{array}{c}-0.17 \\
(-0.86-0.53)\end{array}$ & 0.63 & $\begin{array}{c}-0.18 \\
(-0.87-0.51)\end{array}$ & 0.61 \\
\hline Unstable Relationships & $\begin{array}{c}16 \\
(37.2 \%)\end{array}$ & $\begin{array}{c}0.91 \\
(0.34-1.49)\end{array}$ & 0.003 & $\begin{array}{c}0.20 \\
(-0.44-0.84)\end{array}$ & 0.53 & $\begin{array}{c}0.43 \\
(-0.20-1.06)\end{array}$ & 0.17 & $\begin{array}{c}0.50 \\
(-0.13-1.12)\end{array}$ & 0.12 & $\begin{array}{c}-0.25 \\
(-0.89-0.39)\end{array}$ & 0.43 & $\begin{array}{c}-0.20 \\
(-0.84-0.44)\end{array}$ & 0.54 \\
\hline Identity Disturbance & $\begin{array}{c}33 \\
(76.7 \%)\end{array}$ & $\begin{array}{c}1.20 \\
(0.56-1.83)\end{array}$ & $<0.001$ & $\begin{array}{c}0.77 \\
(0.07-1.47)\end{array}$ & 0.03 & $\begin{array}{c}0.20 \\
(-0.53-0.94)\end{array}$ & 0.58 & $\begin{array}{c}0.04 \\
(-0.70-0.78)\end{array}$ & 0.92 & $\begin{array}{c}-0.19 \\
(-0.93-0.54)\end{array}$ & 0.59 & $\begin{array}{c}-0.16 \\
(-0.90-0.58)\end{array}$ & 0.66 \\
\hline Impulsivity & $\begin{array}{c}28 \\
(65.1 \%)\end{array}$ & $\begin{array}{c}1.39 \\
(0.91-1.88)\end{array}$ & $<0.001$ & $\begin{array}{c}0.68 \\
(0.06-1.29)\end{array}$ & 0.03 & $\begin{array}{c}0.58 \\
(-0.05-1.21)\end{array}$ & 0.07 & $\begin{array}{c}0.31 \\
(-0.33-0.96)\end{array}$ & 0.34 & $\begin{array}{c}-0.64 \\
(-1.15-(- \\
0.12))\end{array}$ & 0.02 & $\begin{array}{c}-0.33 \\
(-0.98-0.31)\end{array}$ & 0.31 \\
\hline Suicidal Behaviour & $\begin{array}{c}36 \\
(83.7 \%)\end{array}$ & $\begin{array}{c}0.44 \\
(-0.39-1.28)\end{array}$ & 0.29 & $\begin{array}{c}0.74 \\
(-0.07-1.55)\end{array}$ & 0.07 & $\begin{array}{c}0.00 \\
(-0.85-0.84)\end{array}$ & 0.997 & $\begin{array}{c}-0.17 \\
(-0.64-0.29)\end{array}$ & 0.45 & $\begin{array}{c}0.07 \\
(-0.77-0.92)\end{array}$ & 0.86 & $\begin{array}{c}0.05 \\
(0.80-0.89)\end{array}$ & 0.91 \\
\hline Affective Instability & $\begin{array}{c}29 \\
(67.4 \%)\end{array}$ & $\begin{array}{c}1.21 \\
(0.66-1.75)\end{array}$ & $<0.001$ & $\begin{array}{c}0.57 \\
(-0.07-1.21)\end{array}$ & 0.08 & $\begin{array}{c}0.74 \\
(0.11-1.36)\end{array}$ & 0.02 & $\begin{array}{c}0.06 \\
(-0.60-0.73)\end{array}$ & 0.85 & $\begin{array}{c}-0.64 \\
(-1.16-(- \\
0.12))\end{array}$ & 0.02 & $\begin{array}{c}-0.28 \\
(-0.94-0.38)\end{array}$ & 0.38 \\
\hline $\begin{array}{l}\text { Chronic Feelings of } \\
\text { Emptiness }\end{array}$ & $\begin{array}{c}36 \\
(83.7 \%)\end{array}$ & $\begin{array}{c}0.18 \\
(-0.66-1.02)\end{array}$ & 0.67 & $\begin{array}{c}0.57 \\
(-0.26-1.39)\end{array}$ & 0.17 & $\begin{array}{c}-0.25 \\
(-1.09-0.59)\end{array}$ & 0.55 & $\begin{array}{c}-0.44 \\
(-1.27-0.40)\end{array}$ & 0.30 & $\begin{array}{c}0.46 \\
(-0.37-1.29)\end{array}$ & 0.27 & $\begin{array}{c}0.26 \\
(-0.58-1.10)\end{array}$ & 0.54 \\
\hline Inappropriate Anger & $\begin{array}{c}20 \\
(46.5 \%)\end{array}$ & $\begin{array}{c}0.68 \\
(0.09-1.26)\end{array}$ & 0.03 & $\begin{array}{c}1.09 \\
(0.56-1.61)\end{array}$ & $<0.001$ & $\begin{array}{c}-0.39 \\
(-1.00-0.22)\end{array}$ & 0.20 & $\begin{array}{c}-0.53 \\
(-1.15-0.10)\end{array}$ & 0.10 & $\begin{array}{c}-0.68 \\
(-1.29-(- \\
0.06))\end{array}$ & 0.03 & $\begin{array}{c}0.19 \\
(-0.43-0.81)\end{array}$ & 0.54 \\
\hline $\begin{array}{c}\text { Transient, Stress- } \\
\text { Related Paranoid } \\
\text { Ideation or Severe } \\
\text { Dissociative Symptoms }\end{array}$ & $\begin{array}{c}22 \\
(51.1 \%)\end{array}$ & $\begin{array}{c}0.83 \\
(0.26-1.40)\end{array}$ & 0.005 & $\begin{array}{c}0.93 \\
(0.37-1.48)\end{array}$ & 0.002 & $\begin{array}{c}-0.38 \\
(-0.99-0.23)\end{array}$ & 0.22 & $\begin{array}{c}-0.02 \\
(-0.64-0.60)\end{array}$ & 0.95 & $\begin{array}{c}-0.13 \\
(-0.75-0.49)\end{array}$ & 0.67 & $\begin{array}{c}-0.15 \\
(-0.78-0.47)\end{array}$ & 0.62 \\
\hline
\end{tabular}

Table 2: The number and percentage of participants endorsing a particular borderline personality disorder symptom and the associations of these individual symptoms with Personality Structure Questionnaire personality fragmentation and with Five-Factor Model personality dimensions. 
Citation: Säämänen T, Voutilainen J, Lahti J, Isometsä E, Heikkinen M, et al. (2016) The Associations of Borderline Personality Disorder Symptoms, Five-Factor Model Personality Dimensions, and Personality Fragmentation among Depressed Inpatients. J Psychiatry 19: 362 doi: $10.4172 / 2378-5756.1000362$

Page 5 of 8

(both endorsed by $83.7 \%$ of the patients) were the BPD symptoms most endorsed in the current study sample. There were no significant group differences between those with and without these BPD symptoms on the assessed personality dimensions, possibly due to low statistical power to detect significant differences because of the low number of patients without these symptoms. No differences were either found between those with or without BPD symptom frantic efforts to avoid abandonment.

The bivariate correlations of FFM personality dimensions and PSQ with SCID-PQ BPD sum-score and with the number of endorsed BPD symptoms are shown in Table 3 and the results of the regression analyses in Table 4 . The significant associations were the following: PSQ personality fragmentation and FFM Neuroticism were positively and FFM Agreeableness negatively correlated with SCID-PQ BPD symptom sum-score. PSQ and Neuroticism were also positively correlated with the number of endorsed BPD symptoms while Agreeableness was not. Extraversion, Openness to Experience and Conscientiousness were not significantly associated with either BPD symptom score.

Also in the regression models adjusted for age, education level and/or CES-D concurrent depressive symptoms, higher PSQ and higher Neuroticism were significantly and independently associated with higher SCID-PQ BPD sum-score and with a higher number of endorsed BPD symptoms. The association of Agreeableness to SCIDPQ BPD sum-score was rendered marginally significant in the adjusted model. These regression models also showed that PSQ personality fragmentation explained the largest amount of variance in SCID-PQ BPD sum-score and in the number of endorsed BPD symptoms in our study sample, followed by Neuroticism (Table 4).

Table 5 shows the results of the Pearson correlation and linear regression analyses on the associations between FFM facet scales and the BPD symptom scores. Higher scores on all Neuroticism facet scales; Anxiety, Depression, Self-Consciousness, Impulsivity, Hostility and Vulnerability were significantly correlated with higher SCIDPQ BPD sum-score and with a higher number of endorsed BPD symptoms. Higher scores on Hostility and Vulnerability remained significant predictors of higher amount of endorsed BPD symptoms and higher SCID-PQ BPD sum-score in regression models adjusted for covariates. In contrast, higher Self-Consciousness was independently associated with the SCID-PQ BPD sum-score, not with the number of endorsed symptoms. Impulsivity, Anxiety, and Depression facets showed no significant associations in the adjusted regression models. Higher scores on Extraversion facet scale Excitement Seeking were correlated with significantly higher SCID-PQ BPD sum-score, but in adjusted models this association was no longer significant. No other significant associations between Extraversion facets and BPD symptom scores were found, and none of the Openness to Experience facets was significantly associated with BPD symptom scores. Furthermore, although the Neuroticism facet scales were the only independent facet scale predictors of BPD symptoms, the amounts of explained variance by each Neuroticism facet of both SCID-PQ BPD sum-score and the number of endorsed BPD symptoms were smaller than for the broad FFM Neuroticism dimension.

\section{Discussion}

In a depressed inpatient sample, higher personality fragmentation and higher Neuroticism were significantly associated with increased BPD symptoms, both when assessed with a more traditional sumscore and with the number of endorsed DSM BPD symptoms. These associations were independent of age, education level and concurrent depressive symptoms. Agreeableness was correlated with BPD symptom sum-score, but showed no significant associations in adjusted regression models. According to effect size estimates, personality fragmentation explained between 38 and $47 \%$ of BPD symptom variance, while the corresponding figure was $14-21 \%$ for Neuroticism. FFM facet scale analyses showed significant associations between multiple different

\begin{tabular}{|c|c|c|c|c|}
\hline Personality Dimension & $\begin{array}{l}\text { Correlation with } \\
\text { SCID-PQ Borderline Personality } \\
\text { Disorder Symptom Sum-Score }\end{array}$ & $p$ & $\begin{array}{l}\text { Correlation with the Number of } \\
\text { Endorsed Borderline Personality } \\
\text { Disorder Symptoms }\end{array}$ & $p$ \\
\hline Personality Fragmentation & 0.76 & $<0.001$ & 0.72 & $<0.001$ \\
\hline Neuroticism & 0.61 & $<0.001$ & 0.57 & $<0.001$ \\
\hline Extraversion & 0.07 & 0.65 & 0.13 & 0.39 \\
\hline Openness to Experience & -0.05 & 0.74 & -0.03 & 0.84 \\
\hline Agreeableness & -0.39 & 0.01 & -0.24 & 0.11 \\
\hline Conscientiousness & -0.19 & 0.23 & -0.09 & 0.57 \\
\hline
\end{tabular}

Table 3: Pearson correlations of Personality Structure Questionnaire and NEO Personality Inventory scales with borderline personality disorder symptoms.

\begin{tabular}{|c|c|c|c|c|c|c|}
\hline \multirow[b]{2}{*}{ Personality Dimension } & \multicolumn{3}{|c|}{ SCID-PQ Borderline Personality Disorder Symptom Sum-Score } & \multicolumn{3}{|c|}{ Number or Endorsed Borderline Personality Disorder Symptoms } \\
\hline & $\beta(95 \% \mathrm{Cl})^{\mathrm{a}, \mathrm{b}}$ & $\mathbf{R}^{2}$ change & $p$-value & $\beta(95 \% \mathrm{Cl})^{\mathrm{d}, \mathrm{e}}$ & $\mathbf{R}^{2}$ change ${ }^{f}$ & $p$-value \\
\hline Personality Fragmentation & $0.70(0.51,0.90)$ & 0.468 & $<0.001$ & $0.64(0.43,0.86)$ & 0.382 & $<0.001$ \\
\hline Neuroticism & $0.55(0.25,0.85)$ & 0.214 & 0.001 & $0.47(0.15,0.80)$ & 0.142 & 0.006 \\
\hline Extraversion & $0.08(-0.21,0.38)$ & 0.007 & 0.57 & $0.17(-0.12,0.45)$ & 0.027 & 0.24 \\
\hline Openness to Experience & $-0.00(-0.30,0.30)$ & 0.000 & 0.99 & $0.01(-0.28,0.30)$ & 0.000 & 0.94 \\
\hline Agreeableness & $-0.28(-0.58,0.02)$ & 0.069 & 0.07 & $-0.12(-0.42,0.19)$ & 0.012 & 0.44 \\
\hline Conscientiousness & $-0.09(-0.40,0.22)$ & 0.007 & 0.56 & $-0.09(-0.38,0.21)$ & 0.007 & 0.55 \\
\hline
\end{tabular}

${ }^{a}$ Adjusted for age and education level; bStandardized regression coefficients $(\beta)$ and their $95 \%$ Confidence intervals $(\mathrm{Cl})$ from linear regression models on the associations of Five-Factor Model and Personality Structure Questionnaire personality dimensions with borderline personality disorder symptom sum-score; ${ }^{\circ} \mathrm{Changes}$ in estimated amount of variance explained in regression models after the inclusion of the personality dimension in question. Age and education level were entered to the model first and the

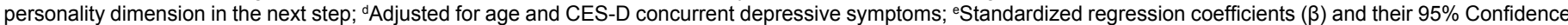
intervals $(\mathrm{Cl})$ from linear regression models on the associations of Five-Factor Model and Personality Structure Questionnaire personality dimensions with the number of endorsed borderline personality disorder symptoms; 'Changes in estimated amount of variance explained in regression models after the inclusion of the personality dimension in question. Age and CES-D depressive symptoms were entered to the model first and the personality dimension in the next step.

Table 4: Linear regression models of personality dimensions predicting borderline personality disorder symptoms. 
Citation: Säämänen T, Voutilainen J, Lahti J, Isometsä E, Heikkinen M, et al. (2016) The Associations of Borderline Personality Disorder Symptoms, Five-Factor Model Personality Dimensions, and Personality Fragmentation among Depressed Inpatients. J Psychiatry 19: 362 doi: $10.4172 / 2378-5756.1000362$

Page 6 of 8

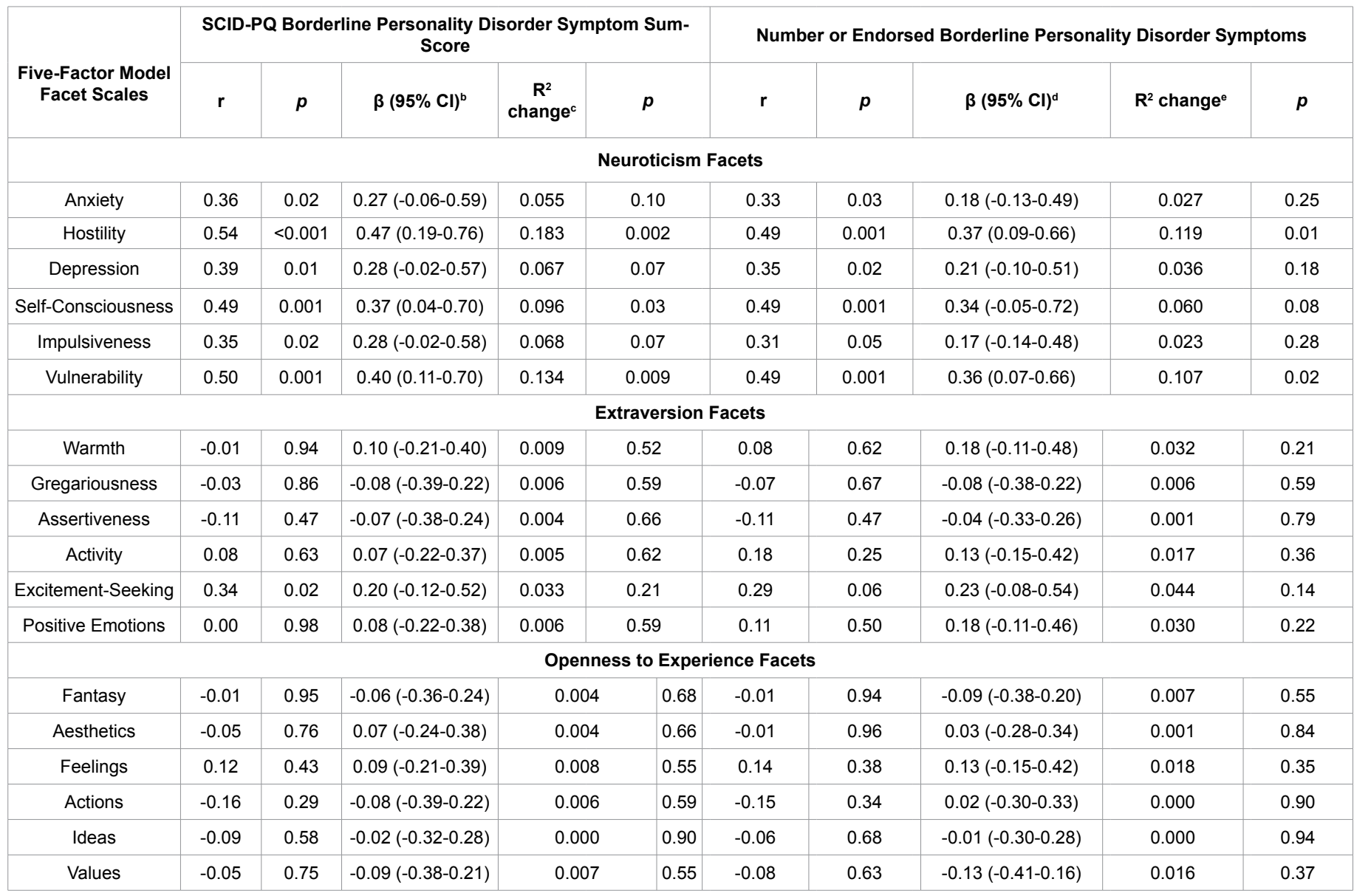

aPearson correlation coefficients between Five-Factor Model facet scale scores and the two borderline personality disorder symptom measures; ${ }^{\circ}$ Standardized regression coefficients $(\beta)$ and their $95 \%$ Confidence intervals $(\mathrm{CI})$ from linear regression models on the associations of Five-Factor Model personality facets with the SCID-PQ borderline personality disorder symptom sum-score, from regression models adjusted for age and education level; ${ }^{\circ} \mathrm{Changes}$ in estimated amount of variance explained in regression models after the inclusion of the personality facet in question. Age and education level were entered to the model first and the personality facet in the next step; dStandardized regression coefficients $(\beta)$ and their $95 \%$ Confidence intervals $(\mathrm{Cl})$ from linear regression models on the associations of Five-Factor Model personality facets with the number of endorsed borderline personality disorder symptoms, from regression models adjusted for age and concurrent depressive symptoms; ${ }^{e} \mathrm{Changes}$ in estimated amount of variance explained in regression models after the inclusion of the personality facet in question. Age and CES-D depressive symptoms were entered to the model first and the personality facet in the next step.

Table 5: Pearson correlation analyses and linear Regression models of Five-Factor Model personality facet scales in predicting borderline personality disorder symptoms.

Neuroticism facets and higher BPD symptoms. Preliminary BPD symptom-level analyses suggested that personality fragmentation and Neuroticism are associated with a wide range of BPD symptoms, while Agreeableness and Extraversion show more specific symptom-level associations.

In accordance with our hypotheses and the findings of Morey et al. [19], we found that FFM Neuroticism increases and Agreeableness decreases further in depressed patients as BPD symptoms increase. However, the associations of Agreeableness were inconsistent. Quite correspondingly, in previous studies, Neuroticism has been the FFM dimension most associated with BPD, while Agreeableness has shown significant but smaller sized effects [17-19]. Also in agreement with hypothesis and previous findings [17-19], Openness to Experience was not associated with BPD symptoms. In contrast to the hypothesis and/or to previous findings $[17,19]$, neither Conscientiousness nor Extraversion were significantly associated with BPD symptoms in our sample. The small sample size or the sample comprising only depressed patients may help to explain the discrepancies between the current and previous findings on Conscientiousness and Extraversion, and the inconsistent effects of Agreeableness. Yet, our findings support the use of dimensional personality traits as one basis for describing personality disorders in diagnostic nomenclature $[1,34,35]$, since Neuroticism shows an independent, significant association with BPD symptoms also among depressed inpatients.

Although high neuroticism and BPD are both strongly associated with MDD [2-5,15,16], our findings suggest that these two more stable personality characteristics also share some features that may differentiate them from the more state-like depression. BPD and Neuroticism are characterized by similar patterns of emotional experience and behavior. BPD symptoms include emotional instability and high levels of negative emotions including chronic feelings of emptiness and episodic dysphoria, irritability and anxiety [1]. Correspondingly, Neuroticism is characterized by high negative affectivity; a trait-like disposition to experience high levels of negative emotions including sadness, depression, anxiety, and anger [13]. Both Neuroticism and BPD are also described by high impulsivity $[1,13]$. Hence, the consistent, specific associations found in current and previous studies between high Neuroticism and BPD may reflect these shared characteristic patterns of 
Citation: Säämänen T, Voutilainen J, Lahti J, Isometsä E, Heikkinen M, et al. (2016) The Associations of Borderline Personality Disorder Symptoms, Five-Factor Model Personality Dimensions, and Personality Fragmentation among Depressed Inpatients. J Psychiatry 19: 362 doi: $10.4172 / 2378-5756.1000362$

Page 7 of 8

emotion processing and behavior. Indeed, our symptom-level analyses suggested that Neuroticism is more strongly associated with BPD symptoms related to anger and impulse control and to a fragmented selfconcept than it is to symptoms related to functioning in interpersonal relationships. Since the vast majority of our depressed inpatient sample endorsed the symptoms of chronic feelings of emptiness and suicidality, we did not have sufficient statistical power to reliably examine their associations with Neuroticism or other personality dimensions.

Agreeableness, in turn, is a personality dimension described by prosocial features such as altruism, trust, modesty, and cooperativeness [13]. Since patients with BPD have more interpersonal problems, more hostile interaction styles and more negative perceptions of others [3638] also in comparison to MDD patients [36], the tentative associations found between low Agreeableness and higher BPD symptoms among depressed inpatients seem logical, particularly, since at the symptomlevel, low Agreeableness was associated with BPD symptoms of inappropriate anger and impulsivity. Interestingly, low Agreeableness was also associated with affective instability, as was high Extraversion. However, all the symptom-level associations were found in group comparisons with small group sizes and with high risks of type I and type II errors and must hence be interpreted with caution.

Findings from facet-scale analyses corresponded to the findings of the broader FFM dimensions: all Neuroticism facets were significantly correlated with higher BPD symptoms, while Openness to Experience and Extraversion facets showed no consistent associations. High Hostility and high Vulnerability were the Neuroticism facets most consistently associated with BPD symptoms in adjusted models. In a previous meta-analysis [17], Depression was the Neuroticism facet most strongly associated with BPD [17], while, corresponding to our findings, each Neuroticism facet showed significant associations. Perhaps due to the high overall depression levels in the current sample, other facets than Depression had stronger associations with BPD symptoms here. Nevertheless, corresponding to previous findings [17], the broad FFM Neuroticism domain explained a larger amount of variance in BPD symptoms than any Neuroticism facet, suggesting it captures variance in BPD psychopathology over and above that captured by the individual facet scales. The lack of associations to Openness to Experience-facets also corresponds with previous findings [17]. In contrast to our findings, previous studies have suggested significant associations of low scores on Extraversion facets Warmth and Positive Emotions with BPD [17]. Possibly these characteristics are more shared than differentiating between MDD and BPD and hence do not associate with BPD symptoms among depressed patients.

The findings of independent associations between higher personality fragmentation and higher BPD symptoms correspond with previous findings in other patient populations that have found personality fragmentation to be most elevated in BPD patients [24]. Personality fragmentation offers an estimate of the combined extent of variability in emotion, behavior and experience of the self across different situations and the level of structural dissociation between these self-states $[21,23,24]$. High personality fragmentation can also be conceptualized as having multiple self-states [21,23,24]. Based on its definition, it thus describes the combined extent of the instability of self-image and affect, dissociation, and identity disturbance characteristics of BPD. Our symptom-level analyses suggested that personality fragmentation may actually be associated with a wider range of BPD symptoms. These include both the related symptoms of identity disturbance, affective instability, and transient, stress-related paranoid ideation/severe dissociative symptoms, and also impulsivity, inappropriate anger and unstable relationships. Hence, personality fragmentation may capture some specific characteristics helpful in identifying individuals with elevated BPD symptoms among patients with comorbid MDD. That personality fragmentation had the largest effect size on BPD symptoms in our study further suggests an important role for personality fragmentation in BPD psychopathology among depressed patients. Personality fragmentation has not previously been included in the dimensional personality assessment systems [34] and has not been as extensively studied as the FFM personality dimensions. It can thus provide a valuable additional approach to the assessment of dimensional personality characteristics associated with BPD.

The limitations of our study include utilizing a small inpatient sample, which can limit the generalizability of our findings to the overall MDD population. Additionally, we assessed self-reported BPD and MDD symptoms, not using stricter diagnostic classifications, limiting the representativeness of the findings to symptom-level associations. Since all the participants were depressed in patients undergoing hospital treatment, their high concurrent depression levels may have induced state-dependent effects on the assessed personality traits and influenced the associations found. Although the particular focus of our study was to assess the specific associations of personality dimensions with BPD symptoms among depressed patients, further studies could examine the same associations among patients with remitted depression. Furthermore, comorbidity with other mental disorders than MDD or bipolar disorder was not accounted for. We neither had FFM facet data for Agreeableness and Conscientiousness. Yet, we used validated questionnaires to assess the FFM personality dimensions, personality fragmentation and BPD symptoms, and our study is among the first to assess which personality dimensions characterize depressed patients with increased BPD symptoms.

\section{Conclusion}

In conclusion, higher personality fragmentation and higher Neuroticism were independently associated with increased BPD symptoms in a depressed inpatient sample. These two personality dimensions may thus informatively describe MDD patients with high levels of BPD symptoms.

\section{Authors' Contributions}

TS and ML participated to conceptualizing the design of the study and to the acquisition of the data, carried out the analyses and interpreted the data, drafted the initial manuscript, and revised the manuscript for intellectual content. JL and EI participated to conceptualizing the design of the study, to the interpretation of the data and to revising the manuscript. JV and $\mathrm{MH}$ contributed to the interpretation of the data and to revising the manuscript. JV also contributed to the acquisition of the data. All authors approved the final manuscript as submitted and agree to be accountable for the integrity of the data.

\section{Acknowledgements}

The authors like to thank Saana Sarparanta for her assistance in data gathering. This study was funded by grants from Academy of Finland and University of Helsinki. The sponsors played no role in the conduct or the design of the study.

\section{References}

1. American Psychiatric Association (2013) Diagnostic and Statistical Manual of Mental Disorders. (5th edn.), American Psychiatric Publishing, Arlington, Virginia.

2. Grant BF, Chou SP, Goldstein RB, Huang B, Stinson FS, et al. (2008) Prevalence, correlates, disability, and comorbidity of DSM-IV borderline personality disorder: results from the Wave 2 National Epidemiologic Survey on 
Citation: Säämänen T, Voutilainen J, Lahti J, Isometsä E, Heikkinen M, et al. (2016) The Associations of Borderline Personality Disorder Symptoms, Five-Factor Model Personality Dimensions, and Personality Fragmentation among Depressed Inpatients. J Psychiatry 19: 362 doi: $10.4172 / 2378-5756.1000362$

Page 8 of 8

Alcohol and Related Conditions J Clin Psychiatry 69: 533-545.

3. Lenzenweger MF, Lane MC, Loranger AW, Kessler RC (2007) DSM-IV personality disorders in the National Comorbidity Survey Replication. Biol Psychiatry 62: 553-564.

4. Johnson DM, Shea MT, Yen S, Battle CL, Zlotnick C, et al. (2003) Gender differences in borderline personality disorder: Findings from the Collaborative Longitudinal Personality Disorders Study. Compr Psychiatry 44: 284-292.

5. Zimmerman M, Mattia J (1999) Axis I diagnostic comorbidity and borderline personality disorder. Compr Psychiatry 40: 245-252.

6. Bolton JM, Robinson J (2010) Population-attributable fractions of Axis I and Axis II mental disorders for suicide attempts: findings from a representative sample of the adult, noninstitutionalized US population. Am J Public Health 100: $2473-2480$.

7. Grilo CM, Sanislow CA, Shea MT, Skodol AE, Stout RL, et al. (2005) Two-year prospective naturalistic study of remission from major depressive disorder as a function of personality disorder comorbidity. J Consult Clin Psychol 73: 78-85.

8. Skodol AE, Grilo CM, Keyes KM, Geier T, Grant BF, et al. (2011) Relationship of personality disorders to the course of major depressive disorder in a nationally representative sample. Am J Psychiatry 168: 257-264.

9. Skodol AE, Gunderson JG, McGlashan TH, Dyck IR, Stout RL, et al. (2002) Functional Impairment in Patients With Schizotypal, Borderline, Avoidant, or Obsessive-Compulsive Personality Disorder. Am J Psychiatry 159: 276-283.

10. Bender DS, Skodol AE, Pagano ME, Dyck IR, Grilo CM, et al. (2006) Prospective assessment of treatment use by patients with personality disorders. Psychiatr Serv 57: 254-257.

11. Gunderson JG, Stout RL, Sanislow CA, Shea MT, McGlashan TH, et al. (2008) New episodes and new onsets of major depression in borderline and other personality disorders. J Affect Disord 111: 40-45.

12. Costa PT, McCrae RR (1992) Normal personality assessment in clinica practice: The NEO Personality Inventory. Psychol Assessment 4: 5-13.

13. McCrae RR, Costa PT Jr (1997) Personality trait structure as a human universal. Am Psychol 52: 509-516.

14. Pulver A, Allik J, Pulkkinen L, Hämäläinen M (1995) A Big Five personality inventory in two non $\square$ Indo $\square$ European languages. Eur J Personality 9: 109-124.

15. Malouff JM, Thorsteinsson EB, Schutte NS (2005) The relationship between the five-factor model of personality and symptoms of clinical disorders: A metaanalysis. J Psychopathol Behav 27: 101-114

16. Kotov R, Gamez W, Schmidt F, Watson D (2010) Linking "big" personality traits to anxiety, depressive, and substance use disorders: A meta-analysis. Psychol Bull 136: 768-821.

17. Samuel DB, Widiger TA (2008) A meta-analytic review of the relationships between the five-factor model and DSM-IV-TR personality disorders: A facet level analysis. Clin Psychol Rev 28: 1326-1342.

18. Saulsman LM, Page AC (2004) The five-factor model and personality disorde empirical literature: A meta-analytic review. Clin Psychol Rev 23: 1055-1085.

19. Morey LC, Gunderson JG, Quigley BD, Shea MT, Skodol AE, et al. (2002) The representation of borderline, avoidant, obsessive-compulsive, and schizotypal personality disorders by the five-factor model. J Pers Disord 16: 215-234.

20. Lieb K, Zanarini MC, Schmahl C, Linehan MM, Bohus M (2004) Borderline personality disorder. Lancet 364: 453-461.

21. Ryle A (1997) The structure and development of borderline personality disorder: a proposed model. Br J Psychiatry 170: 82-87.

22. Bennett D, Ryle A (2005) The characteristic features of common borderline states: a pilot study using the States Description Procedure. Clin Psycho Psychot 12: 58-66.

23. Ryle A (2007) Investigating the phenomenology of Borderline Personality Disorder with the States Description Procedure: Clinical implications. Clin Psychol Psychot 14: 329-341.

24. Pollock PH, Broadbent M, Clarke S, Dorrian A, Ryle A (2001) The personality structure questionnaire (PSQ): a measure of the multiple self states model of identity disturbance in cognitive analytic therapy. Clin Psychol Psychot 8: 5972.

25. Wildgoose A, Waller G, Clarke S, Reid A (2000) Psychiatric symptomatology in borderline and other personality disorders: dissociation and fragmentation as mediators. J Nerv Ment Dis 188: 757-763.

26. Beck AT, Steer RA, Brown GK (1996) Manual for beck depression inventory II (BDI-II). Psychology Corporation. San Antonio, Texas.

27. Radloff LS (1977) The CES-D Scale: A self-report depression scale for research in the general population. Appl Psych Meas 1: 385-401

28. Hirschfeld RM, Williams JB, Spitzer RL, Calabrese JR, Flynn L, et al. (2000) Development and Validation of a Screening Instrument for Bipolar Spectrum Disorder: The Mood Disorder Questionnaire. Am J Psychiatry 157: 1873-1875

29. Chanen AM, Jovev M, Djaja D, McDougall E, Yuen HP, et al. (2008) Screening for borderline personality disorder in outpatient youth. J Pers Disord 22: 353 364.

30. Korzekwa MI, Dell PF, Links PS, Thabane L, Webb SP (2008) Estimating the prevalence of borderline personality disorder in psychiatric outpatients using a two-phase procedure. Compr Psychiatry 49: 380-386.

31. Costa PT, McCrae RR (1985) The NEO Personality Inventory Manual. Psychological Assessment Resources. Odessa, Florida.

32. Blom G (1958) Statistical estimates and transformed beta-variables. Wiley New York.

33. Bedford A, Davies F, Tibbles J (2009) The Personality Structure Questionnaire (PSQ): a cross-validation with a large clinical sample. Clin Psychol Psychother 16: 77-81.

34. Hopwood CJ, Thomas KM, Markon KE, Wright AGC, Krueger RF (2012) DSM5 personality traits and DSM-IV personality disorders. J Abnorm Psychol 121 424-432.

35. Melartin TK, Haukka J, Rytsälä HJ, Jylhä PJ, Isometsä ET (2010) Categorica and dimensional stability of comorbid personality disorder symptoms in DSM-IV major depressive disorder: a prospective study. J Clin Psychiatry 71: 287-295.

36. Barnow S, Stopsack M, Grabe HJ, Meinke C, Spitzer C, et al. (2009) Interpersonal evaluation bias in borderline personality disorder. Behav Res Ther 47: 359-365

37. Sadikaj G, Moskowitz D, Russell JJ, Zuroff DC, Paris J (2013) Quarrelsome behavior in borderline personality disorder: Influence of behavioral and affective reactivity to perceptions of others. J Abnorm Psychol 122: 195-207.

38. Sanislow CA, Grilo CM, Morey LC, Bender DS, Skodol AE, et al. (2002) Confirmatory Factor Analysis of DSM-IV Criteria for Borderline Personality Disorder: Findings From the Collaborative Longitudinal Personality Disorders Study. Am J Psychiatry 159: 284-290. 